\title{
Wavelet-Based Power Network Disturbance Identifying Part I: Theory and Method
}

\author{
Gang Chen ${ }^{1, a^{*}}$, Ming Tang ${ }^{1, b}$, Lijie Ding $^{1, c}$, Hua Zhang ${ }^{1, d}$ \\ ${ }^{1} 24$ Qinghua Road, Qingyang District, Chengdu, Sichuan, 610072, CHINA \\ agangchen08@gmail.com, btangming_king@163.com, ‘ding_lijie@163.com, \\ dzhanghua002@163.com
}

Keywords: WAMS, wavelet transform, wavelet coefficient, disturbance identifying.

\begin{abstract}
It is important that control center operators be alerted to system disturbances, including where, when and what disturbance occurs, so that proper anticipatory actions can be promptly taken if necessary, avoiding oscillation spreads in the power network. In this paper, the wavelet multi-resolution analysis based method is proposed to identify power system disturbances. Energy of wavelet coefficients are used as a criterion to choose optimal wavelet function and decomposition scale, which are then used for obtaining the maximum wavelet coefficients by identifying the frequency signals from wide area measurement system (WAMS). The maximum wavelet coefficients are then selected to be the indicators for disturbance identifying. The detailed procedure and effectiveness of the proposed method is demonstrated by simulations of a 10-machine 39-bus system.
\end{abstract}

\section{Introduction}

The synchronized phasor measurement units (PMUs) are able to measure and record the rotor angle, the frequency, the magnitude and phase of voltage and current of power systems. The measured data are sent to the phasor data concentrator (PDC) through high speed internet. Accordingly, a wide area measurement system (WAMS) is constructed based on the PMUs, PDC and internet. Thereby, the operational condition of the power grid can be monitored continuously and the dynamics of the power system can be recorded[1].

One of the most important application of WAMS is using the data recorded by PMUs to identify disturbances in power systems, including fault diagnosis[2], power quality disturbance identifying[3] as well as power network disturbance identifying[4]. The power network disturbance identifying mainly focus on disturbances leading to serious accidents in power system, such as cutting machine, load shedding, etc.

Wavelet transform (WT) is a powerful and systematic way of analyzing the abrupt-changing feature of signals, belonging to a type of time-frequency-domain analysis[5]. Using WT, the frequency features of the analyzed signal could be located in time domain. Thus, as a promising tool, WT deserves a thorough study when it is applied to WAMS-based disturbance analysis. In[6], for the first time, the WT was introduced to disturbance identification, detecting the time when disturbance occurred.

In this paper, the theory of a wavelet-based power network disturbance identifying method is described. First, the WT-based multi-resolution analysis is introduced in Section 1. As the key part of the method, the theory of power network disturbance identifying method, based on WT, is described in Section 2. The case study is given in Section 3. The conclusion is discussed in Section 4.

\section{WT-Based Multi-Resolution Analysis (MRA)}

WT is a partial time-frequency-domain analysis method, the frequency and time window of which can be changed, obtaining both the time and frequency domain information at the same time. Using WT, signals can be decomposed into several sub-spaces in different resolutions, so that signals with different frequencies are displayed in the different sub-spaces, revealing their characteristics clearly. 
For any signal $x(t) \in L^{2}(R)$, it can be decoupled by WT as the following equation:

$$
x(t)=\sum_{K} a_{J}(k) \varphi_{J, k}(t)+\sum_{j+1}^{J} \sum_{K} d_{j}(k) \psi_{j, k}(t)
$$

where $a_{J}(k)$ and $d_{j}(k)$ are the Scaling coefficients (SCs, also called approximation), and Wavelet coefficients (WCs, also called detail), respectively. $\varphi_{J, k}(t)$ is the Scaling function and $\psi_{j, k}(t)$ is the Wavelet function.

Mallat algorithm provides a convenient way of applying WT, especially in using this fast algorithm for time-frequency analysis. Given the signals, their features can be obtained once we have the two-scale equation dual channel filter coefficients $\left\{h_{0}(k)\right\}$ and $\left\{h_{1}(k)\right\}$, without knowing the functions of Scaling function $\varphi_{J, k}(t)$ and Wavelet function $\psi_{j, k}(t)$.

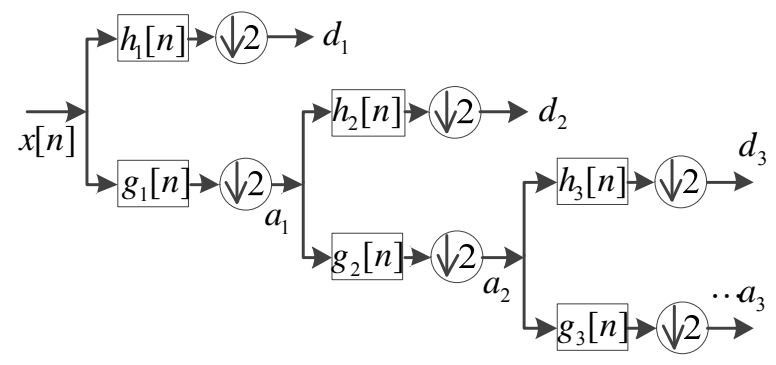

Fig. 1 Procedure of WT-based MRA

For a signal $x[n]$, the procedure of WT-based MRA can be shown in Fig. 1. Using WT-based MRA, the SCs and WCs can be easily obtained. It should be noted that WCs contain high-frequency characteristics of the signal, which can be used to detect disturbance accidents by selecting an appropriate wavelet function and a decomposition level. In addition, WT also has data compression, signal noise, signal reconstruction and non-redundant decomposition of other unique advantages, which is very useful in signal analysis and processing.

\section{Power Network Disturbance Identifying Based on MRA}

When power network disturbances, including load switching and load loss, etc., the frequencies at each bus changes in the system of the same trend with different specific values, causing certain spatial and temporal distribution characteristics[8]. The dynamic distribution characteristics of frequency means that, when disturbances occur, the frequency of power system will change, and the closer to the disturbance source, the faster the frequency changes, as well as the greater the amount of change. Therefore, the frequency signal,which has uniform reference of $50 \mathrm{~Hz}$, is a natural input signal for disturbance identifying.

The energy of WCs is chosen as the criterion to choose optimal wavelet function and decomposition scale. Using the following equation, the energy contained in WCs can be obtained:

$$
E_{i}=\sum_{j=1}^{n_{i}}\left|d_{i j}\right|^{2}
$$

where $i$ is the scale; $j$ is the number of points contained in each detail.

According to Eq. 2, the WC's energy of the disturbance can be obtained by summing up the energy of signals, since there are several frequency responses at the PMU locations. Therefore, the selection of wavelet function should obtain the maximum WC's energy for specific disturbances.

Having the wavelet function, we can get the total energy of all decomposition levels by:

$$
E=\sum_{i=1}^{N} E_{i}
$$


where $N$ denotes the decouple scale; $i$ is the total energy of all decomposition levels. The decomposition scale can be selected by having the maximum energy according to Eq. 3 .

Usually, the highest WC appears at the corresponding abrupt-changing point of the analyzed signal, while identifying transient signals using WT-Based MRA. Therefore, the time of the highest WC appears can be estimated as the moment of disturbance occurs. Moreover, according to the simple principle that the closer the observation position is, the higher WC can be obtained. The PMU sub-station where the highest WC is observed could be estimated as the location closest to the location where disturbance occurs. Therefore, we choose the highest WC as disturbance identification index.

\section{Case Study}

In this section, the IEEE 39 bus system[7] is selected to illustrate the using of the method proposed in this paper. The scheme of IEEE New England39-bus system is illustrated in Figure 2. PMUs are located at Bus 3, 12, 19, 22, 29 and Bus 39, which have been marked in red in Fig. 2.

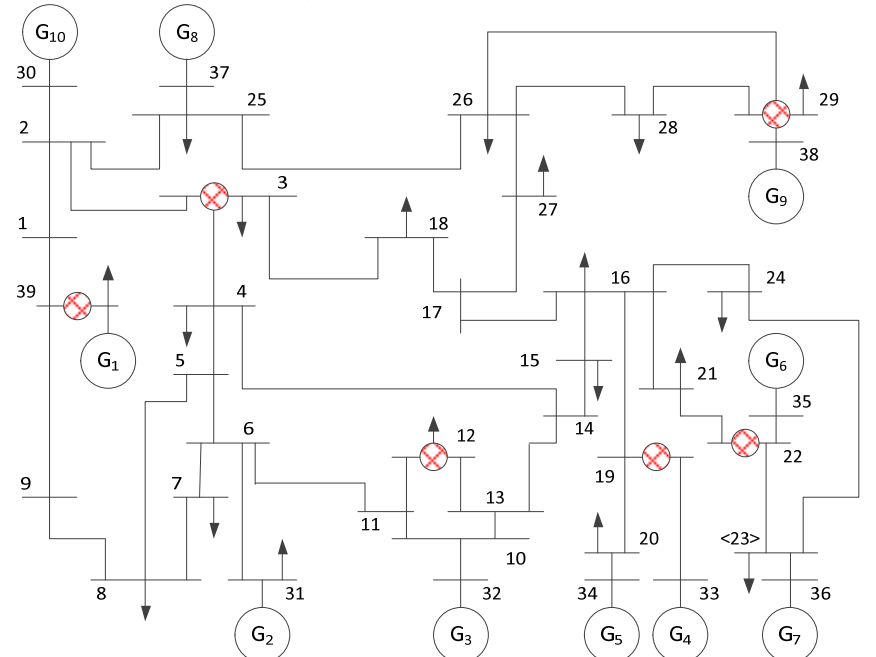

Fig. 2 the IEEE New England39-bus system

In this paper, Order 2-11 Daubechies wavelets are taken into consideration. And the maximum scale is chosen to be 6 . Six disturbances are simulated: D1, generator loss at G4; D2, generator loss at G5; D3, load loss at Bus 16; D4, load loss at load bus 20; D5, load increase at Bus 16; D6, load increase at Bus 20. Frequencies at the Buses with PMUs are identified with MRA, obtaining the each energy of WC for each disturbance, which are shown in Table 1. It can be seen from Table 1, the maximum energy is concentrated in the first row, therefore, the choice of wavelet function is "db2".

Table 1 The selection of wavelet function for 10-machine 39-bus power system

\begin{tabular}{ccccccccccc}
\hline wavelet function & Db2 & Db3 & Db4 & Db5 & Db6 & Db7 & Db8 & Db9 & Db10 & Db11 \\
\hline D1 & $\mathbf{1 . 1 7 9}$ & 0.761 & 0.673 & 0.714 & 0.395 & 0.637 & 0.881 & 1.009 & 0.836 & 0.7 \\
D2 & $\mathbf{0 . 4 5 4}$ & 0.315 & 0.228 & 0.259 & 0.134 & 0.182 & 0.281 & 0.369 & 0.294 & 0.22 \\
D3 & $\mathbf{0 . 1 6 4}$ & 0.031 & 0.079 & 0.181 & 0.096 & 0.092 & 0.136 & 0.092 & 0.073 & 0.121 \\
D4 & $\mathbf{0 . 6 7 8}$ & 0.436 & 0.481 & 0.678 & 0.521 & 0.505 & 0.659 & 0.634 & 0.544 & 0.527 \\
D5 & $\mathbf{0 . 3 6 2}$ & 0.248 & 0.194 & 0.23 & 0.108 & 0.146 & 0.238 & 0.311 & 0.237 & 0.185 \\
D6 & 1.133 & 0.726 & 0.686 & 0.68 & 0.58 & 0.672 & 0.78 & 1.403 & $\mathbf{1 . 7 6 1}$ & 1.728 \\
\hline
\end{tabular}

According to the selected wavelet function of"db2", the energy of WCs using diffenent decomposition scales are shown in Table 2. It can be seen from Table 2 that the energy of WCs is concentrated in the 5 th floor, so the choice of decomposition scale is 5. 
Table 2 The selection of scale for 10-machine 39-bus power system

\begin{tabular}{ccccccc}
\hline Scale & 1 & 2 & 3 & 4 & $\mathbf{5}$ & 6 \\
\hline D1 & 0.018 & 0.038 & 0.021 & 0.351 & $\mathbf{0 . 4 6 2}$ & 0 \\
D2 & 0.003 & 0.009 & 0.007 & 0.118 & $\mathbf{0 . 1 4 9}$ & 0 \\
D3 & 0.001 & 0.002 & 0.001 & 0.034 & $\mathbf{0 . 0 4 4}$ & 0 \\
D4 & 0.009 & 0.016 & 0.009 & 0.161 & $\mathbf{0 . 1 7 9}$ & 0 \\
D5 & 0.001 & 0.004 & 0.003 & 0.061 & $\mathbf{0 . 0 8 9}$ & 0 \\
D6 & 0.016 & 0.046 & 0.042 & 0.362 & $\mathbf{0 . 4 6 2}$ & 0 \\
\hline
\end{tabular}

The frequencis recorded by PMUs are indentifying using the wavelat function "db2" for the scale of 5. The results are shown in Fig. 3 and Fig. 4, in which the discrete wavelet coefficients in the Figures are connected by straight lines, in order to show details more clearly.

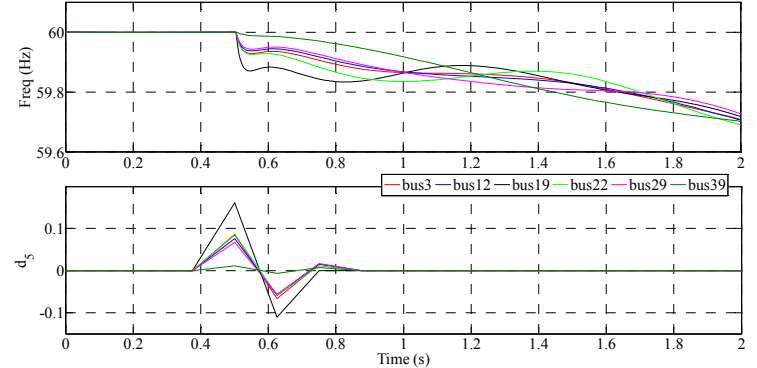

Fig. 3 Wavelet coefficients for frequency responses to generator loss of G5

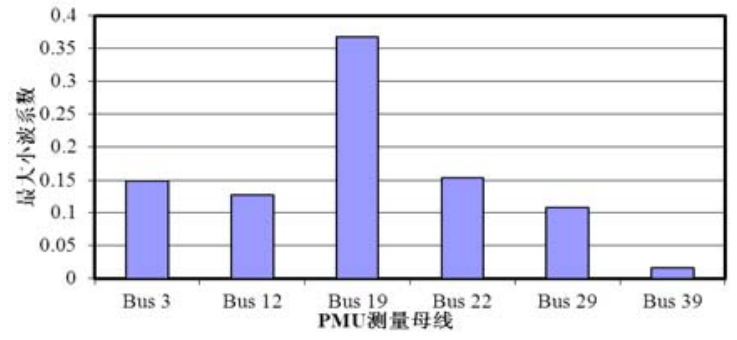

Fig. 4 Maximum of wavelet coefficients at measuring Buses for generation loss at G5

As shown in Fig. 3, when the disturbance of genertor loss at G5 at the time of $0.5 \mathrm{~s}$, the largest wavelet coefficients obtained by the observation buses appear at $0.5 \mathrm{~s}$, indicating that the occurrence of the disturbance is at $0.5 \mathrm{~s}$. In Fig. 4 , it can be seen that the maximum wavelet coefficient appears at Bus 19, which is the nearest one from G5. Therefore, the Bus with the largest wavelet coefficient can be estimated as the disturbance source.

The results of load loss and increse are shown in Fig. 5 and Fig. 6. It can be seen from Fig. 5 and Fig. 6 that, the largest wavelet coefficients still appear at the moment of the disturbance occurred. The nearest Bus for disturbance source also has the largest wavelet coefficients and the furthest bus 39 has the minimum value, with the removal of the disturbance generator was the same conclusion.

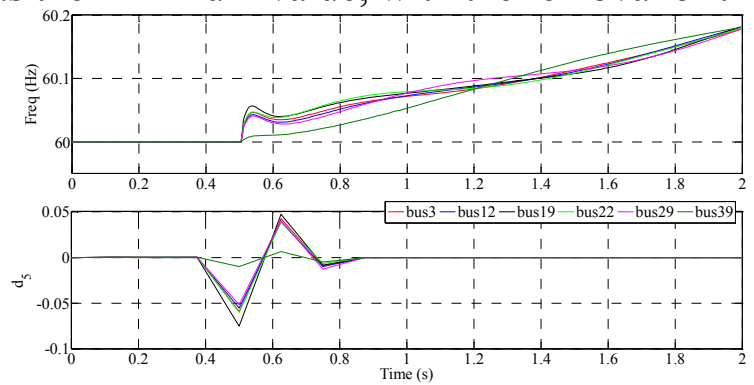

Fig. 5 Wavelet coefficients for frequency responses to load loss at Bus 16

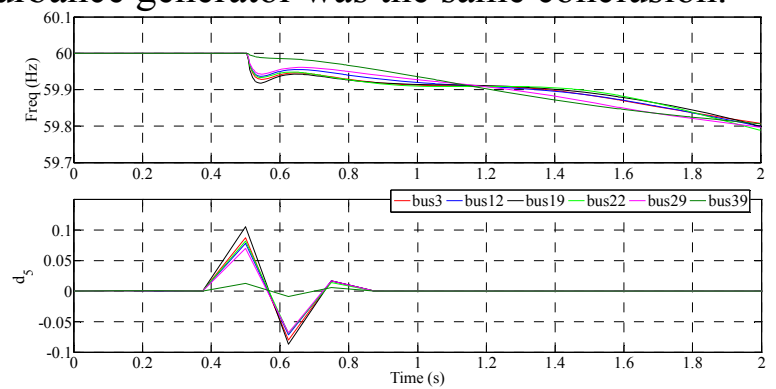

Fig. 6 Wavelet coefficients for frequency responses to load increase at Bus 16

\section{Conclusions}

In this paper, an wavelet-based power network disturbance identifying method is presentd. The method of selection of input signal, wavelet function, decomposition scale and indicator are proposed. The maximum of wavelet coefficients are chosen to indentify the occuring time and source of disturbance. Based on the data recorded by WAMS, it is easy to identify disturbances, which can help operators to take timely and appropriate actions to eliminate the impact of disturbance.

In part II, the application of the method in large scale power system will be proposed[8]. 


\section{References}

[1] LUO Jian-yu, WANG Xiao-ying, Lu Ting-rui, et al. Application of Power Network Real-time Monitoring System Based on Wide-area Measurement Technology [J]. Automation of Electric Power System, 2003(24):78-80. .

[2] LAN Hua, SUN Chuan-meng, WANG Yun-ran, et al. Distributed Fault Diagnosis in Power System Based on Bayesian Networks [J]. Electrical Measurement \& Instrumnenation, 2012, 49(563): $1-5$.

[3] DUGAN R. C., MEGRANGHAN M. F., Benty H. W. Electrical Power System Quality [M]. New York: MC Graw-Hill, 1996.

[4] DONG J. Y., ZUO J., WANG L., et al. Analysis of Power System Disturbances Based on Wide-area Frequency Measurements [C]. IEEE PES General Meeting, Tampa, FL, USA, 2007.

[5] MEI K. J., ROVNYAK S. M., and ONG C. M. Clustering-based Dynamic Event Location Using Wide-area Phasor Measurements [J]. IEEE Transactions on Power Systems, 2008, 23(2): 673-679.

[6] MEI K. J., ROVNYAK S. M., and ONG C. M. Dynamic Event Detection Using Wavelet Analysis [C]. IEEE PES General Meeting, Lafayette, 2006:1-7.

[7] ZHANG Y. C., MARKHAM P., XIA T., et al. Wide-Area Frequency Monitoring Network (FNET) Architecture and Applications [J]. IEEE Trans. Smart Grid, 2010, 1(2): 159-167.

[8] PAI M. A. Energy Function Analysis for Power System Stability [M]. Boston: Kluwer Academic Publishers, 1989.

[9] CHEN Gang, ZHOU Bo, ZHANG Hua, et al. Wavelet-Based Power Network Disturbance Identifying Part II: Application in Large Scale Power System, 2015 6th International Conference on Advances in Circuits, Electronics and Micro-Electronics(CENICS 2015), Xi'an, China. 